\title{
AN UNUSUAL MONOTONICITY THEOREM WITH APPLICATIONS
}

\author{
J. MALÝ, D. PREISS AND L. ZAJÍČEK
}

(Communicated by R. Daniel Mauldin)

\begin{abstract}
In the article an unusual monotonicity theorem is proved. It easily implies some usual monotonicity theorems which deal with preponderant and symmetrical derivatives. We also obtain some results concerning one-sided densities of arbitrary linear sets, which are closely related to an O'Malley theorem.
\end{abstract}

1. Introduction. In an investigation of the behavior of "typical" continuous functions an unusual monotonicity theorem is useful, namely the main theorem, Theorem 1, of our article for the case of a continuous $f$ and $S=\varnothing$. It was formulated and proved in a rather complicated manner by the third named author. The present generalized theorem and its simple proof are results of collaboration of all three authors. As immediate consequences we obtain some usual monotonicity theorems which deal with preponderant and symmetrical derivates. In particular we obtain a solution of Query 166 posed in the Real Analysis Exchange by L. Larson [4, p. 295]. We also easily obtain some results concerning one-sided densities of arbitrary sets which are closely related to the interesting O'Malley theorem [6]. An application to the behavior of typical continuous functions will appear in a subsequent article.

In the following, $\mu\left(\mu^{*}, \mu_{*}\right)$ stands for the Lebesgue measure (outer Lebesgue measure, inner Lebesgue measure) on the real line $R$. The characteristic function of a set $A \subset R$ is denoted by $\chi_{A}$. For measurable sets $M \subset R$ we denote by $d^{+}(M, x)$ and $d_{-}(M, x)$ the right upper and the left lower densities of $M$ at $x$, respectively. The right lower inner density of an arbitrary set $M \subset R$ at $x$ is defined as

$$
d_{i,+}(M, x)=\liminf _{h \rightarrow 0+} h^{-1} \mu_{*}(M \cap(x, x+h)) .
$$

The symbol $h_{n} \searrow 0$ means that $h_{1} \geq h_{2} \geq \cdots>0$ and $h_{n} \rightarrow 0$.

\section{The main theorem.}

THEOREM 1. Suppose that $f$ is a real-valued function defined on an interval $[a, b]$ and that $\alpha \in(0,1)$. Suppose further that there is a set $S \subset(a, b)$ such that the following conditions hold.

(A) For every $x \in(a, b) \backslash S$,

(A1) there is $y \in(x, b]$ such that $\mu^{*}\{z \in(x, y): f(z)>f(x)\} \geq \alpha(y-x)$, or

Received by the editors November 10, 1986.

1980 Mathematics Subject ('lassification (1985 Revision). Primary 26A24.

Key words and phrases. Monotonicity theorems, preponderant derivates, symmetrical derivates, one-sided densities. 
(A2) there is $y \in[a, x)$ such that $\mu^{*}\{z \in(y, x): f(z)>f(x)\}<\alpha(x-y)$.

(B) For every $x \in[a, b)$ and every $c<f(x)$, there is $y \in(x, b]$ such that $\mu^{*}\{z \in(x, y): f(z)>c\} \geq \alpha(y-x)$.

(C) For every $x \in(a, b]$ and every $d>f(x)$, there is $y \in[a, x)$ such that $\mu^{*}\{z \in(y, x): f(z)>d\}<\alpha(x-y)$.

(D) $f(S)$ contains no interval.

Then $f(b) \geq f(a)$.

ProOF. Suppose to the contrary that $f(b)<f(a)$. Let $v \in(f(b), f(a)) \backslash f(S)$. For $t \in[a, b]$ put

$$
g(t)=-\alpha t+\mu^{*}\{z \in(a, t): f(z)>v\} .
$$

Since $g$ is continuous, there exists $m=\max \{g(t): t \in[a, b]\}$. Put $x=$ $\sup \{t \in[a, b]: g(t)=m\}$; clearly $g(x)=m$. We shall distinguish the following three possible cases.

(i) $f(x)>v$. Since $f(b)<v, x \in[a, b)$ and condition (B) with $c=v$ implies that there is $y \in(x, b]$ such that $g(y)-g(x)=-\alpha(y-x)+\mu^{*}\{z \in(x, y): f(z)>v\} \geq 0$. Consequently, $g(y) \geq m$ and $y>x$; a contradiction.

(ii) $f(x)<v$. Since $f(a)>v, x \in(a, b]$ and condition (C) with $d=v$ implies that there is $y \in[a, x)$ such that $g(y)-g(x)=\alpha(x-y)-\mu^{*}\{z \in(y, x): f(z)>$ $v\}>0$. Consequently, $g(y)>m$; a contradiction.

(iii) $f(x)=v$. Then $x \in(a, b) \backslash S$. If (A1) holds, there is $y \in(x, b]$ such that $g(y)-g(x)=-\alpha(y-x)+\mu^{*}\{z \in(x, y): f(z)>f(x)\} \geq 0$. Consequently $g(y) \geq m, y>x$; a contradiction. Finally, if (A2) holds, there is $y \in[a, x)$ such that $g(y)-g(x)=\alpha(x-y)-\mu^{*}\{z \in(y, x): f(z)>f(x)\}>0$. Consequently, $g(y)>m$. This is a contradiction which completes the proof.

NoTES. (1) In Theorem 1 we can, of course, replace $\mu^{*}$ by $\mu_{*}$, since the proof works also after this change.

(2) As the example $a=0, b=0, f(x)=\chi_{\{0\} \cup[1 / 2,1)}(x)+x / 2, \alpha=1 / 2, S=\varnothing$ shows, it is not possible to replace " $<\alpha(x-y)$ " by " $\leq \alpha(x-y)$ " in (C).

(3) It is easy to see that the assertion of Theorem 1 holds if we write " $>\alpha(y-x)$ " instead of " $\geq \alpha(y-x)$ " in (A1) and (B), and at the same time " $\leq \alpha(x-y)$ " instead of " $<\alpha(x-y)$ " in (A2) and (C). The point is that we then define $x$ in the proof by $x=\inf \{t \in[a, b]: g(t)=m\}$. The modified conditions will be labeled by $(\tilde{\mathrm{A}})$, $(\widetilde{\mathrm{B}})$, and $(\widetilde{\mathrm{C}})$.

(4) In condition (A1) it is not possible to write $f(z) \geq f(x)$ instead of $f(z)>$ $f(x)$. To see this fact, it is sufficient to consider the function $f=-g$, where $g$ is the well-known Cantor singular function, to set $\alpha=1 / 2$, and to choose $S$ as the set of all points of $[0,1]$ which are bilaterally accumulation points of the Cantor ternary set. On the other hand, we can make this change if we replace condition (D) of Theorem 1 by a slightly stronger condition.

PROPOSITION 1. In the assumptions of Theorem 1, if we replace condition (A1) by condition

$\left(\mathrm{A} 1^{*}\right)$ there is $y \in(x, b]$ such that

$$
\mu^{*}\{z \in(x, y): f(z) \geq f(x)\} \geq \alpha(y-x)
$$


and condition $(\mathrm{D})$ by condition

$\left(\mathrm{D}^{*}\right) I \backslash f(S)$ is uncountable for any interval $I$, then we can again conclude that $f(b) \geq f(a)$.

ProOF. Put $\widetilde{S}=S \cup A$, where $A$ is the set of all points $x \in[a, b)$ for which there is $y \in(x, b]$ such that

$$
\mu^{*}\{z \in(x, y): f(z) \geq f(x)\} \geq \alpha(y-x)>\mu^{*}\{z \in(x, y): f(z)>f(x)\} .
$$

Then assumptions (A), (B), (C) of Theorem 1 hold for the set $\widetilde{S}$, and thus it is sufficient to prove that $f(\widetilde{S})$ contains no interval. To prove this it is sufficient to show that $f(A)$ is countable. Let $I$ be the system of all open subintervals of $(0,1)$ with rational endpoints. It is easy to see that for any $u \in f(A)$ there is $I \in I$ such that the nonincreasing function $g_{I}(t)=\mu^{*}\{z \in I: f(z)>t\}$ is discontinuous at $u$. Consequently, $f(A)$ is countable and Proposition 1 is proved.

3. Applications to one-sided densities of linear sets. If we apply Theorem 1 to $f=\chi_{M}$ and $S=(a, b)$ (or Proposition 1 to $f=\chi_{M}$ and $S=\varnothing$ ), we immediately obtain the following corollary.

COROLlARY 1. Let $\alpha \in(0,1)$ and let $M \subset[a, b]$ be an arbitrary set for which $a \in M$. Suppose that

(A) for any $x \in M \cap[a, b)$, there is $y \in(x, b]$ such that $\mu^{*}(M \cap(x, y)) \geq \alpha(y-x)$, and

(B) for any $x \in(a, b] \backslash M$, there is $y \in[a, x)$ such that $\mu^{*}(M \cap(y, x))<\alpha(x-y)$. Then $b \in M$.

The above statement can be immediately reformulated in the following way.

COROLlARY 2. Let $\alpha \in(0,1)$ and let $M \subset[a, b]$ be an arbitrary set such that $a \in M$ and $b \notin M$. Suppose that for any $x \in M \cap[a, b)$ there is $y \in(x, b]$ such that $\mu^{*}(M \cap(x, y)) \geq \alpha(y-x)$. Then there exists a point $z \in(a, b] \backslash M$ such that for any $y \in[a, z)$ we have $\mu^{*}(M \cap(y, z)) \geq \alpha(z-y)$.

From Corollary 2 we easily obtain the following statement which involves conditions of local nature.

COROLlaRY 3. Let $\alpha \in(0,1)$ and let $\varnothing \neq M \subset(a, b)$ not be a connected set. Suppose that for any $x \in M$ there exists a sequence $h_{n} \searrow 0$ such that

$$
\mu^{*}\left(M \cap\left(x, x+h_{n}\right)\right) \geq \alpha h_{n} .
$$

Then there exists $x \in(a, b) \backslash M$ and $\delta>0$ such that $\mu^{*}(M \cap(x-h, x)) \geq \alpha h$ for any $0<h<\delta$.

PROOF. There exist points $a<a_{1}<b_{1}<b, a_{1} \in M, b_{1} \notin M$. Now it is sufficient to apply Corollary 2 to $\left[a_{1}, b_{1}\right]$ and $M_{1}=M \cap\left[a_{1}, b_{1}\right]$.

Corollary 3 immediately implies the following proposition which is similar to an interesting result of O'Malley [6].

COROLlaRY 4. Let $\beta \in(0,1)$ and let $\varnothing \neq M \subset(a, b)$ be a measurable set which is not connected. Let $d^{+}(M, x)>\beta$ for any $x \in M$. Then there exists a point $z \in(a, b) \backslash M$ for which $d_{-}(M, z) \geq \beta$. 
If we apply the variant of Theorem 1, mentioned in Note (3) after Theorem 1 , to $\chi_{M}$ and $S=(a, b)$ we subsequently obtain Corollary $1^{*}$, Corollary $2^{*}$, and Corollary $3^{*}$, which we shall state explicitly.

COROllary $3^{*}$. Let $\alpha \in(0,1)$ and let $\varnothing \neq M \subset(a, b)$ not be a connected set. Suppose that for any $x \in M$ there exists a sequence $h_{n} \searrow 0$ such that

$$
\mu^{*}\left(M \cap\left(x, x+h_{n}\right)\right)>\alpha h_{n} .
$$

Then there exist $x \in(a, b) \backslash M$ and $\delta>0$ such that $\mu^{*}(M \cap(x-h, x))>\alpha h$ for any $0<h<\delta$.

The following example shows that in Corollary 3 it is not possible to write " $\mu^{*}(M \cap(x-h, x))>\alpha h$ " instead of " $\mu^{*}(M \cap(x-h, x)) \geq \alpha h$."

EXAMPLE 1. Let $a=0, b=1$, and $\alpha \in(0,1)$. Let $F \subset[0,1]$ be the Cantor ternary set and let $\left\{\left(a_{n}, b_{n}\right)\right\}_{n=1}^{\infty}$ be all components of $(0,1) \backslash F$. Put

$$
M=\bigcup_{n=1}^{\infty}\left[b_{n}-\alpha\left(b_{n}-a_{n}\right), b_{n}\right] .
$$

It is easy to see that for any $x \in M$ there exists a sequence $h_{n} \searrow 0$ such that $\mu\left(M \cap\left(x, x+h_{n}\right)\right) \geq \alpha h_{n}$, and for any $x \in(0,1) \backslash M$ there exists a sequence $h_{n} \searrow 0$ such that $\mu\left(\left(x-h_{n}, x\right) \backslash M\right) \geq(1-\alpha) h_{n}$.

4. Applications to monotonicity theorems involving preponderant or symmetrical derivates. There exist several nonequivalent definitions of preponderant notions (cf. $[\mathbf{2}, \mathbf{5}, \mathbf{1}])$. We shall consider here two old possibilities and a new one which leads to the weakest notion of a preponderant limit. Using new preponderant notions we can state in a very simple form the usual monotonicity theorem which immediately follows from our theorem, Theorem 1.

Let $M \subset R$ and $a \in R$ be given. We shall say that

(i) $M$ is (s)-right preponderant (strongly right preponderant) at $a$ if $d_{i,+}(M, a)>$ $1 / 2$,

(ii) $M$ is (w)-right preponderant (weakly right preponderant) at $a$ if there is $\delta>0$ such that $\mu_{*}(M \cap(a, a+h))>h / 2$ for $0<h<\delta$, and

(iii) $M$ is (W)-right preponderant (very weakly right preponderant) at $a$ if there is $\delta>0$ such that $\mu_{*}(M \cap(a, a+h)) \geq h / 2$ for $0<h<\delta$.

In the following, let $\square$ be any of the symbols (s), (w), and (W). The notion of $\square$-left preponderantness is defined in the obvious way. If $M$ is simultaneously $\square$-right and $\square$-left preponderant at $a$, we say that $M$ is $\square$-preponderant at $a$. $M$ is clearly (w)-preponderant ((W)-preponderant) at $a$ iff there is $\delta>0$ such that $\mu_{*}(M \cap(x, y))>\frac{1}{2}(y-x)\left(\mu_{*}(M \cap(x, y)) \geq \frac{1}{2}(y-x)\right)$ whenever $x \leq a \leq y$ and $0<y-x<\delta$.

If $f$ is a function defined on a subset of $R$ we put

$$
\begin{aligned}
& \limsup _{x \rightarrow a} \square \operatorname{pr} f(x)=\inf \{y \in R:\{x: f(x) \leq y\} \text { is } \square \text {-preponderant at } a\}, \\
& \liminf _{x \rightarrow a} \square \operatorname{pr} f(x)=\sup \{y \in R:\{x: f(x) \geq y\} \text { is } \square \text {-preponderant at } a\} .
\end{aligned}
$$

The notions

$$
\underset{x \rightarrow a^{+}}{\limsup } \square \operatorname{pr} f(x), \quad \liminf _{x \rightarrow a^{+}} \square \operatorname{pr} f(x), \quad \underset{x \rightarrow a^{-}}{\limsup } \square \operatorname{pr} f(x)
$$


are defined in the obvious way using the notions of $\square$-right and $\square$-left preponderantness of a set at a point. tions

The extreme $\square$-preponderant derivates $\bar{D}_{\square \mathrm{pr}} f, \underline{D}_{\square \mathrm{pr}} f$ are defined by the equa-

$$
\begin{aligned}
& \bar{D}_{\square \mathrm{pr}} f(x)=\limsup _{y \rightarrow x} \frac{f(y)-f(x)}{y-x}, \\
& \underline{D}_{\square \mathrm{pr}} f(x)=\liminf _{y \rightarrow x} \frac{f(y)-f(x)}{y-x} .
\end{aligned}
$$

The $\square$-preponderant derivates $D_{\square \mathrm{pr}}^{+} f, D_{+\square \mathrm{pr}} f, D_{\square \mathrm{pr}}^{-} f, D_{-\square \mathrm{pr}} f$ are defined quite similarly.

The lower symmetric derivate of $f$ at $x$ is defined as

$$
\underline{D}_{\text {sym }} f(x)=\liminf _{h \rightarrow 0+} \frac{f(x+h)-f(x-h)}{2 h} .
$$

Notes. (5) Obviously

$$
\begin{aligned}
& \limsup _{x \rightarrow a} \square \mathrm{pr} f(x)=\max \left(\limsup _{x \rightarrow a+} \square \mathrm{pr} f(x), \limsup _{x \rightarrow a-} \operatorname{spr}_{\mathrm{pr}} f(x)\right), \\
& \limsup _{x \rightarrow a}(\mathrm{~W}) \operatorname{pr} f(x) \leq \limsup _{x \rightarrow a}(\mathrm{w}) \mathrm{pr} f(x) \leq \limsup _{x \rightarrow a}(\mathrm{~s}) \mathrm{pr} f(x),
\end{aligned}
$$

and

$$
\liminf _{x \rightarrow a}(\mathrm{~s}) \mathrm{pr} f(x) \leq \liminf _{x \rightarrow a}(\mathrm{w}) \mathrm{pr} f(x) \leq \liminf _{x \rightarrow a}(\mathrm{w}) \mathrm{pr} f(x) .
$$

(6) It is not difficult to show (cf. the proof of Lemma 2) that if $M$ is (W)preponderant at $a$ then $R \backslash M$ is not (W)-preponderant at $a$. This observation yields the inequality

$$
\liminf _{x \rightarrow a}(\mathrm{~W}) \operatorname{pr} f(x) \leq \limsup _{x \rightarrow a}(\mathrm{~W}) \mathrm{pr} f(x) .
$$

We shall need the following simple lemmas.

LEMMA 1. Let $f$ be a function defined on a subset of $R$ and let $a \in R$. Then the following assertions hold.

(i) If there exists $\lim _{x \rightarrow a} g(x) \in R$, then

$$
\begin{aligned}
& \limsup _{x \rightarrow a+}(\mathrm{W}) \mathrm{pr}(f+g)(x)=\limsup _{x \rightarrow a+}(\mathrm{W}) \mathrm{pr} f(x)+\lim _{x \rightarrow a} g(x), \\
& \limsup _{x \rightarrow a_{-}}(\mathrm{W}) \mathrm{pr}(f+g)(x)=\limsup _{x \rightarrow a-}(\mathrm{W}) \mathrm{pr} f(x)+\lim _{x \rightarrow a} g(x) .
\end{aligned}
$$

(ii)

$$
\limsup _{x \rightarrow a+}(\mathrm{W}) \operatorname{pr} f(x) \geq \alpha \quad\left(\limsup _{x \rightarrow a+}(\mathrm{w}) \mathrm{pr} f(x) \geq \alpha\right)
$$

iff for all $\beta<\alpha$ there is a sequence $h_{n} \searrow 0$ such that $\mu^{*}\left\{y \in\left(x, x+h_{n}\right): f(y)>\right.$ $\beta\}>h_{n} / 2\left(\mu^{*}\left\{y \in\left(x, x+h_{n}\right): f(y)>\beta\right\} \geq h_{n} / 2\right)$. Similarly

$$
\liminf _{x \rightarrow a-}(\mathrm{W}) \mathrm{pr} f(x) \leq \alpha \quad\left(\liminf _{x \rightarrow a-}(\mathrm{w}) \mathrm{pr} f(x) \leq \alpha\right)
$$


iff for all $\beta>\alpha$ there is a sequence $h_{n} \searrow 0$ such that $\mu^{*}\left\{y \in\left(x-h_{n}, x\right): f(y)<\right.$ $\beta\}>h_{n} / 2\left(\mu^{*}\left\{y \in\left(x-h_{n}, x\right): f(y)<\beta\right\} \geq h_{n} / 2\right)$.

(iii)

$$
\bar{D}_{(\mathrm{W}) \mathrm{pr}} f(x)=\max \left(D_{(\mathrm{W}) \mathrm{pr}}^{+} f(x), D_{(\mathrm{W}) \mathrm{pr}}^{-} f(x)\right)
$$

and

$$
\bar{D}_{(\mathrm{w}) \mathrm{pr}} f(x)=\max \left(D_{(\mathrm{w}) \mathrm{pr}}^{+} f(x), D_{(\mathrm{w}) \mathrm{pr}}^{-} f(x)\right) .
$$

(iv) $D_{(\mathrm{w}) \mathrm{pr}}^{+} f(x) \geq \alpha\left(D_{(\mathrm{w}) \mathrm{pr}}^{+} f(x) \geq \alpha\right)$ iff for all $\beta<\alpha$ there is a sequence $h_{n} \searrow 0$ such that

$$
\begin{gathered}
\mu^{*}\left\{y \in\left(x, x+h_{n}\right): \frac{f(y)-f(x)}{y-x}>\beta\right\}>h_{n} / 2 \\
\left(\mu^{*}\left\{y \in\left(x, x+h_{n}\right): \frac{f(y)-f(x)}{y-x}>\beta\right\} \geq h_{n} / 2\right) .
\end{gathered}
$$

Similarly $D_{(\mathrm{w}) \mathrm{pr}}^{-} f(x) \geq \alpha\left(D_{(\mathrm{w}) \mathrm{pr}}^{-} f(x) \geq \alpha\right)$ iff for all $\beta<\alpha$ there is a sequence $h_{n} \searrow 0$ such that

$$
\begin{gathered}
\mu^{*}\left\{y \in\left(x-h_{n}, x\right): \frac{f(y)-f(x)}{y-x}>\beta\right\}>h_{n} / 2 \\
\left(\mu^{*}\left\{y \in\left(x-h_{n}, x\right): \frac{f(y)-f(x)}{y-x}>\beta\right\} \geq h_{n} / 2\right) .
\end{gathered}
$$

(v) If $h(x)=f(x)+c x$, then $\bar{D}_{(\mathrm{w}) \mathrm{pr}} h(x)=\bar{D}_{(\mathrm{W}) \mathrm{pr}} f(x)+c, \bar{D}_{(\mathrm{w}) \mathrm{pr}} h(x)=$ $\bar{D}_{(\mathrm{w}) \mathrm{pr}} f(x)+c$ and $\underline{D}_{\mathrm{sym}} h(x)=\underline{D}_{\mathrm{sym}} f(x)+c$.

PROOF. It is sufficient to use the definitions in a straightforward way.

LEMMA 2. Let $f$ be a Lebesgue measurable function defined on $(a, b), x \in(a, b)$, and $\underline{D}_{\mathrm{sym}} f(x) \geq 0$. Then $\bar{D}_{(\mathrm{W}) \mathrm{pr}} f(x) \geq 0$.

Proof. Choose $\varepsilon>0$ and put $g(x)=f(x)+\varepsilon x$. Then by Lemma $1(\mathrm{v})$, $\underline{D}_{\text {sym }} g(x)>\varepsilon / 2$ and we can therefore find a $\Delta>0$ such that $g(x+h)>g(x-h)$ for $0<h<\Delta$. For $0<\delta<\Delta$ put $H_{1}^{\delta}=\{h \in(0, \delta): g(x+h)>g(x)\}, H_{2}^{\delta}=\{h \in$ $(0, \delta): g(x-h)<g(x)\}, u_{1}(\delta)=\mu\left(H_{1}^{\delta}\right)$ and $u_{2}(\delta)=\mu\left(H_{2}^{\delta}\right)$. Since, clearly, $(0, \delta) \subset$ $H_{1}^{\delta} \cup H_{2}^{\delta}$, we have $u_{1}(\delta)+u_{2}(\delta) \geq \delta$ and, consequently, $\max \left(u_{1}(\delta), u_{2}(\delta)\right) \geq \delta / 2$ for $\delta \in(0, \Delta)$. Since, for $i \in\{1,2\},\left(u_{i}\right)^{\prime}(\delta)=\chi_{\{h: g(x+h)<g(x)\}}(\delta)$ for almost all $\delta \in(0, \Delta)$, there is no right neighborhood of 0 on which $u_{i}(\delta)=\delta / 2$. Consequently, there is a sequence $\delta_{n} \searrow 0$, and $i \in\{1,2\}$, such that $u_{i}\left(\delta_{n}\right)>\delta_{n} / 2$. By Lemma 1(iii), (iv) this implies that $\bar{D}_{(\mathrm{w}) \mathrm{pr}} g(x) \geq 0$. By Lemma 1 (v) we obtain $\bar{D}_{(\mathrm{W}) \mathrm{pr}} f(x) \geq-\varepsilon$. Since $\varepsilon>0$ was an arbitrary number, we conclude that $\bar{D}_{(\mathrm{W}) \mathrm{pr}} f(x) \geq 0$.

THEOREM 2. Let $g$ be a real function defined on $(c, d)$ such that

(i) for any $x \in(c, d)$

$$
\liminf _{y \rightarrow x-}(\mathrm{W}) \operatorname{pr} g(y) \leq g(x) \leq \limsup _{y \rightarrow x+}(\mathrm{W}) \mathrm{pr} g(y),
$$


(ii) $\bar{D}_{(\mathrm{W}) \mathrm{pr}} g(x) \geq 0$ at all points $x \in(c, d) \backslash S$, where $S$ is a countable set. Then $f$ is nondecreasing on $(c, d)$.

PROOF. Choose arbitrary points $c<a<b<d$ and $\varepsilon>0$. Put $\alpha=1 / 2$ and $f(x)=g(x)+\varepsilon x$. By (i) and Lemma $1(\mathrm{i})$, we have

$$
\liminf _{y \rightarrow x-}(\mathrm{W}) \operatorname{pr} f(y) \leq f(x) \leq \limsup _{y \rightarrow x+}(\mathrm{W}) \operatorname{pr} f(y)
$$

for all $x \in(c, d)$. On account of Lemma 1(ii) this immediately implies that $f$ satisfies conditions (B) and (C) of Theorem 1.

By Lemma $1(\mathrm{v}), \bar{D}_{(\mathrm{W}) \mathrm{pr}} f(x) \geq \varepsilon>0$ for $x \in(c, d) \backslash S$, which implies by Lemma 1(iii), (iv) that also condition (A) of Theorem 1 is satisfied. Consequently, Theorem 1 implies $g(b)+\varepsilon b=f(b) \geq f(a)=g(a)+\varepsilon a$. Letting $\varepsilon \rightarrow 0$ we obtain $g(b) \geq g(a)$ which completes the proof.

Notes. (7) The main result of [3] immediately implies that there is a residual set $F$ in the space of all continuous functions on $[a, b]$ such that for every $f \in F$ we have $\bar{D}_{(\mathrm{s}) \mathrm{pr}} f(x)=+\infty$ and $\underline{D}_{(\mathrm{s}) \mathrm{pr}} f(x)=-\infty$ at all points $x \in(a, b)$. Hence there is no chance to prove an analogue of Theorem 2 for upper strong preponderant derivates.

(8) If we replace $(\mathrm{W})$ by $(\mathrm{w})$ at all three places in Theorem 2 , we then obtain a statement which is false. In fact, it is sufficient to put $c=0, d=1$ and to consider $g=\chi_{M}$, where $M$ is the set from Example 1 (for $\alpha=1 / 2$ ). On the other hand, we have the following

PROPOSITION 2. Let $g$ be a Lebesgue measurable function on $(c, d)$ such that

(i) for any $x \in(c, d)$

$$
\liminf _{y \rightarrow x-}(\mathrm{W}) \operatorname{pr} g(y) \leq g(x) \leq \limsup _{y \rightarrow x+}(\mathrm{W}) \operatorname{pr} g(y),
$$

(ii) $\bar{D}_{(\mathrm{w}) \mathrm{pr}} g(x) \geq 0$ at all points $x \in(c, d) \backslash S$, where $S$ is a countable set. Then $f$ is nondecreasing on $(c, d)$.

PrOOF. Let $a, b, \varepsilon, f, \alpha$ be as in the proof of Theorem 2 . It is sufficient to verify condition ( $\tilde{\mathrm{A}})$ from Note (3), after Theorem 1. Choose $x \in(a, b) \backslash S$. By Lemma $1(\mathrm{v}), \bar{D}_{(\mathrm{w}) \mathrm{pr}} f(x) \geq \varepsilon$ holds. Therefore we can, by Lemma 1 (iv), find $h$ such that either

$$
0<h<x-a \text { and } \mu\{y \in(x-h, x): f(y)<f(x)+\varepsilon(y-x) / 2\} \geq h / 2
$$

or

$$
0<h<b-x \text { and } \mu\{y \in(x, x+h): f(y)>f(x)+\varepsilon(y-x) / 2\} \geq h / 2 .
$$

In the former case, condition $(\tilde{\mathrm{A}})$ is obviously satisifed. In the latter case we shall distinguish two possibilities.

If $f(x+h)>f(x)$, then by the hypotheses of Proposition 2 and Lemma 1(ii), it is possible to find $0<k<b-(x+h)$ such that $\mu\{y \in(x+h, x+h+k): f(y)>$ $f(x)\}>k / 2$. Therefore,

$$
\begin{aligned}
\mu\{y \in & (x, x+h+k): f(y)>f(x)\} \\
\geq & \mu\{y \in(x, x+h): f(y)>f(x)+\varepsilon(y-x) / 2\} \\
& +\mu\{y \in(x+h, x+h+k): f(y)>f(x)\} \\
> & h / 2+k / 2=(h+k) / 2 .
\end{aligned}
$$


If $f(x+h) \leq f(x)$, then, by the hypotheses of Proposition 2 and Lemma 1(ii), we can find $0<k<h$ such that $\mu\{y \in(x+h-k, x+h): f(y)<f(x)+\varepsilon / 3\}>k / 2$ and $f(x)+\varepsilon(y-x) / 2>f(x)+\varepsilon h / 3$ for $y \in(x+h-k, x+h)$. It follows that

$$
\mu\{y \in(x+h-k, x+h): f(y)>f(x)+\varepsilon(y-x) / 2\}<k / 2 .
$$

Thus

$$
\begin{aligned}
\mu\{y \in & (x, x+h-k): f(y)>f(x)\} \\
> & \mu\{y \in(x, x+h-k): f(y)>f(x)+\varepsilon(y-x) / 2\} \\
= & \mu\{y \in(x, x+h): f(y)>f(x)+\varepsilon(y-x) / 2\} \\
& -\mu\{y \in(x+h-k, x+h): f(y)>f(x)+\varepsilon(y-x) / 2\} \\
> & h / 2-k / 2=(h-k) / 2 .
\end{aligned}
$$

Therefore condition $(\tilde{\mathrm{A}})$ is again verified which completes the proof.

Using Lemma 2, we see that Theorem 2 immediately implies the following proposition which answers affirmatively Query 166, posed by L. Larson [4].

PROPOSITION 3. Let $f$ be an approximately continuous function on $(a, b)$ such that $\underline{D}_{\text {sym }} f(x) \geq 0$ except a countable set. Then $f$ is nondecreasing on $(a, b)$.

The following proposition may also be of some interest.

PROPOSITION 4. Let $\beta>0$ and let $f$ be an approximately continuous function on $(c, d)$ such that the right upper density of $\{y: f(y) \geq f(x)\}$ at $x$ is at least $\beta$ for all $x \in(c, d) \backslash S$, where $S$ is a countable set. Then $f$ is nondecreasing on $(c, d)$.

PROOF. It is sufficient to apply Proposition 1 for $c<a<b<d$ and $\alpha=\beta / 2$.

\section{BIBLIOGRAPHY}

1. A. M. Bruckner, Some observations about Denjoy's preponderant derivative, Bull. Math. Soc. Sci. Math. R. S. Roumanie 21 (69) (1977), 1-10.

2. A. Denjoy, Sur une propriete des fonctions derivees, Enseign. Math. 18 (1916), 320-328.

3. V. Jarník, Sur la dérivabilité des fonctions continues, Publ. Faculté Sci. Univ. Charles 129 (1934).

4. L. Larson, Query 166, Real Anal. Exchange 9 (1983-84), 295.

5. J. L. Leonard, Some conditions implying the monotonicity of a real function, Rev. Roumanie Math. Pures Appl. 17 (1972), 757-780.

6. R. J. O'Malley, A density property and applications, Trans. Amer. Math. Soc. 199 (1974), 75-87.

MATEMATICKO-FyZIKÁlNí FAKULTA UNIVERSity KARLOVY, SOKOLOVSKÁ 83, 18600 Praha 8, CZEChOSLOVAKIA 